\title{
Philosophiques
}

\section{Remerciements à nos lecteurs et lectrices}

Volume 17, numéro 2, automne 1990

URI : https://id.erudit.org/iderudit/027138ar

DOI : https://doi.org/10.7202/027138ar

Aller au sommaire du numéro

Éditeur(s)

Société de philosophie du Québec

ISSN

0316-2923 (imprimé)

1492-1391 (numérique)

Découvrir la revue

Citer ce document

(1990). Remerciements à nos lecteurs et lectrices. Philosophiques, 17(2),

234-237. https://doi.org/10.7202/027138ar d'utilisation que vous pouvez consulter en ligne.

https://apropos.erudit.org/fr/usagers/politique-dutilisation/ 


\section{Remerciements à nos lecteurs et lectrices}

Depuis le $1^{\text {er }}$ janvier 1986 , les personnes suivantes ont accepté d'évaluer des textes pour Pbilosophiques. Nous les en remercions vivement.

ALLARD, Gérard, Collège de Sainte-Foy, philosophie.

ALLARD, Guy, Université de Montréal, études médiévales.

AUROUX, Sylvain, Université de Paris VII, histoire des théories linguis. tiques.

AYOUB, Josiane, Université du Québec à Montréal, philosophie.

BEAUSOLEIL, Jocelyn, Université du Québec à Montréal, sciences de l'éducation.

BÉGIN, Luc, Université du Québec à Montréal, philosophie.

BÉLANGER, Jean, Université du Québec à Montréal, psychologie.

BELlEMARE, Pierre, Université du Québec à Trois-Rivières, philosophie. BODÉUS, Richard, Université de Montréal, philosophie.

BORDELEAU, Léo-Paul, Université d'Ottawa, philosophie.

BOUCHARD, Guy, Université Laval, philosophie.

BRAYBROOKE, David, Dalhousie University, philosophie.

CANTIN, Serge, Université du Québec à Trois-Rivières, philosophie.

CHARRON, Ghyslain, Université d'Ottawa, philosophie.

CHENÉ, Adèle, Université de Montréal, sciences de l'éducation.

COUTURE, Jocelyne, Université du Québec à Montréal, philosophie.

COUTURIER, Fernand, Université du Québec à Montréal, philosophie.

CRAGG, Wesley, Université Laurentienne de Sudbury, philosophie.

CROTEAU, Jacques, Université d'Ottawa, philosophie.

DESPLAND, Michel, Université Concordia, sciences religieuses.

DION, Michel, Université du Québec à Trois-Rivières, théologie.

DUCHESNEAU, François, Université de Montréal, philosophie.

DUMAS, Brigitte, Université de Montréal, sociologie.

DUMOUCHEL, Paul, Université du Québec à Montréal, philosophie.

FOISY, Suzanne, Université du Québec à Trois-Rivières, philosophie. 
FRENCH, Stanley, Université Concordia, philosophie.

GAGNON, Maurice, Université de Sherbrooke, philosophie.

GARCEAU, Benoît, Université d'Ottawa, philosophie.

GENEST, Olivette, Université de Montréal, théologie.

GIROUX, Laurent, Université de Sherbrooke, philosophie.

GRONDIN, Jean, Université d'Ottawa, philosophie.

GUÉDON, Jean-Claude, Université de Montréal, histoire et sociopolitique des sciences.

HURKA, Tom, University of Calgary, Philosophie.

KaUFMANN, J. Nicolas, Université du Québec à Trois-Rivières, philosophie.

KING-FARLOW, John, University of Alberta, philosophie.

KLIMOV, Alexis, Université du Québec à Trois-Rivières, philosophie.

KNEE, Philip, Université Laval, philosophie.

LACHARITÉ, Normand, Université du Québec à Montréal, philosophie.

LAFRANCE, Guy, Université d'Ottawa, philosophie.

LAFRANCE, Yvon, Université d'Ottawa, philosophie.

LAMONTAGNE, Claude, Université d'Ottawa, psychologie.

LAMOUREUX, Diane, Université Laval, sciences politiques.

LANE, Gilles, Université de Montréal, philosophie.

LATRAVERSE, François, Université du Québec à Montréal, philosophie.

LAURIER, Daniel, Université de Montréal, philosophie.

LÉONARD, Jean-François, Université du Québec à Montréal, sciences politiques.

LEPAGE, François, Université de Montréal, philosophie.

LEROUX, Jean, Université d'Ottawa, philosophie.

LEROUX, Georges, Université du Québec à Montréal, philosophie.

LETOCHA, Danièle, Université d'Ottawa, philosophie.

LEVESQUE, Claude, Université de Montréal, philosophie.

LUC, Laurent-Paul, Université de Sherbrooke, philosophie.

MARCIL-LACOSTE, Louise, Université de Montréal, philosophie.

McCALL, Storrs, Université McGill, philosophie.

McDONELL, Donald, Université d'Ottawa, philosophie.

MENDENHALL, Vance, Université d'Ottawa, philosophie.

MEUNIER, Jean-Guy, Université du Québec à Montréal, philosophie.

MIGUELEZ, Roberto, Université d'Ottawa, sociologie. 
MILOT, Louise, Université Laval, études littéraires.

MONETTE, Lise, Université du Québec à Montréal, philosophie.

MULLETT, Sheila, Université Concordia, philosophie.

NADEAU, Robert, Université du Québec à Montréal, philosophie.

NAUD, Julien, Université du Québec à Trois-Rivières, philosophie.

NIELSEN, Kai, University of Calgary, philosophie.

PANACCIO, Claude, Université du Québec à Trois-Rivières, philosophie.

PARMENTIER, Francis, Université du Québec à Trois-Rivières, français.

PELLERIN, René, Collège Laflèche, philosophie.

PESTIAU, Joseph, Collège de Saint-Laurent, philosophie.

PICHÉ, Claude, Université de Montréal, philosophie.

PROULX, Carole, Collège de Trois-Rivières, philosophie.

RACINE, Luc, Université de Montréal, sociologie.

RENAULT, Marc, Université du Québec à Trois-Rivières, philosophie.

RIOUX, Bertrand, Université de Montréal, philosophie.

ROBERT, Serge, Université du Québec à Montréal, philosophie.

ROULEAU, Jean-Paul, Université Laval, sociologie.

ROUSSEAU, Louis, Université du Québec à Montréal, sciences de la religion.

ROY, David-J., Institut de recherches cliniques de Montréal.

ROY, Jean, Université de Montréal, philosophie.

RUSE, Michael, Guelph University, philosophie.

SCHLEIFER, Michael, Université du Québec à Montréal, sciences de l'éducation.

TERRASSE, Jean, Université McGill, langue et littérature françaises.

THEAU, Jean, Université d'Ottawa, philosophie.

TOURNIER, François, Université Laval, philosophie.

VACHET, André, Université d'Ottawa, sciences politiques.

VAILLANCOURT, Jean-Guy, Université de Montréal, sociologie.

VANDERVEKEN, Daniel, Université du Québec à Trois-Rivières, philosophie.

Juillet 1990 . 